\title{
VERIFICATION OF AUTOMATA MODELS FOR CLINICAL DECISION SUPPORT SYSTEMS
}

\author{
Grigorii Shovkoplias ${ }^{1}$, Ivan Smirnov ${ }^{1}$, Mark Tkachenko ${ }^{1}$, Natalia Gusarova ${ }^{1}$, Aleksandra Vatian ${ }^{1}$, \\ Anatoly Shalyto ${ }^{1}$ and Rajdeep Niyogi ${ }^{2}$ \\ ${ }^{1}$ ITMO University, Sankt-Petersburg 197101, Russia \\ ${ }^{2}$ Indian Institute of Technology Roorkee. 247667, India
}

\begin{abstract}
In the treatment of any disease, the doctor must strictly follow the clinical protocol. However, this document cannot be fully considered as a graph diagram of the algorithm of the doctor's actions, since most of the transition rules in it cannot be implemented without the involvement of the doctor's knowledge. So, based on the clinical protocol, it is necessary to create a clinical decision support system (CDSS). Existing CDSSs do not allow describing the clinical protocol as a sequence of actions. This drawback can be eliminated by using the automata model as a CDSS inference engine, since the terminology of the treatment process itself leads to a natural thought - to model this process in states, i.e. use an automata approach. At the same time, it is vital to ensure the extensibility of the automata model, which will allow among other things for taking into account when choosing drugs any possible adverse reactions to drugs already prescribed.

The article discusses the process of development and verification of an automata model as an inference engine for CDSS. The process, based on the Automata approach and performed offline, uses a sequence of refinements, which allows you to get a more detailed model that reflects the typical options for the doctor. In addition, with each replacement or addition of the drug, online testing of the model is performed, during which compatibility with the already prescribed drugs and the patient's history is checked. The developed process is applied to the construction and verification of CDSS for the management of patients with multiple sclerosis.
\end{abstract}

\section{KEYWORDS}

Automata, Clinical Decision Support System, Verification, Multiple Sclerosis

\section{INTRODUCTION}

Clinical decision making is a fundamental aspect of the work of the clinician, which has a direct impact on the course of treatment of the patient. Clinical decision making is defined as a contextual, continuous, and evolving process through which data are collected, interpreted, and evaluated to make informed choices [Tiffen]. The set of factors that determine the context is very wide [Johansen]; it includes the patient's health state, available treatment options (including equipment, medicines, etc.), the clinical experience of the doctor, the patient's personal characteristics, and the level of accessible healthcare environment in general. At the same time, the guiding document for the doctor is the official clinical recommendations for the management of a specific disease. Thus, when making clinical decisions, the clinician must take into account a complex set of conditions, and to support this process, the use of clinical decision support systems (CDSS) is effective [Horsky, Bologva].

An official source [Clinica] defines CDSS as a system that provides clinicians with knowledge reasonably filtered or presented at the right time to improve health and medical care. Accordingly, these definitions cover a wide range of healthcare support systems that have been announced as projects or are already on the market (see reviews [Rawson, Reis]. A detailed analysis showed that most of CDSS implement the following functionalities individually or in combination: support for individual diagnostic procedures (for example, automatic analysis of radiographs, CT and MRI images), statistical evaluation of indicators and access to medical calculators, reference functions (for example, checking with drug capacities, structured access to health services); record indicators with remote monitoring of patients; manual recording of tasks (during treatment in the clinic). However, they do not allow describing the clinical protocol as a formalized sequence of actions. 
Significant advantages in this regard are provided by the use of the automata model [Khoussainov, Khoussainov, Polikarpova] in the framework of CDSS. The concept of formal methods is widely used in healthcare (see review [Bonfanti]). A substantial analysis of the terminology used to describe the clinical process suggests that the state in the automata model and the state of the patient in the clinical process are close not only terminologically, but also conceptually. In particular, the archetype of State Machine as a basic concept is recommended for use in the openEHR medical standard [openEHR]. Note that modern means of supporting automated programming provide ample opportunities for modeling decision-making processes [Mitkin]. Many authors [Bonfanti, Banach] consider the verification of automata models (and formal modeling methods in general) a basic advantage for modeling medical processes, where the safety requirement is critically important. In [ten Teije, Groot, M'ery] models of medical protocol are proposed, but they consider the protocol as purely formal, i.e. do not imply variability in the behavior of the doctor in relation to a particular patient.

Thus, the introduction of an automated approach to the development and verification of CDSS to support the clinical process is an important and urgent task. In this article, this problem is solved by the example of the management of patients suffering from multiple sclerosis [NINDS] - the most common autoimmune disorder of the central nervous system, resulting in physical, mental, and psychiatric problems up to disability.

\section{PROBLEMS OF USING AUTOMATA MODELS IN CDSS}

Although studies of multiple sclerosis are conducted on a broad front, a radical cure for this disease still does not exist [NINDS]. The management scheme for a patient with multiple sclerosis [19] includes primary diagnosis, assessment of the degree of disability, treatment in the acute stage, as well as the prescription of drugs that can change the course of multiple sclerosis. Each stage is described by a set of recommendations in natural language, accompanied by a detailed commentary, and contains so called "Algorithms for the management of the patient", which are models (schemes) of the corresponding stage of the clinical process. A detailed analysis showed that this documentation structure is characteristic not only for multiple sclerosis, but also for other diseases.

Unfortunately, these models, although called algorithms, are not such in the formal sense, since they contain different types of logical discrepancies and inconsistencies [ten Teije, Groot, M’ery, Gusarova, Vatian]. Many authors [ten Teije, Groot, M'ery] propose a rigorous formalization of clinical protocols as a whole, however, such a path rarely appears to be acceptable in real clinical practice. For the practical use of clinical protocols, it is necessary by default to "load" an extensive and dynamically changing context associated with the knowledge and experience of a clinician. At the same time, the more detailed the fragment of the clinical protocol, the more formally its model and corresponding context can be set. These circumstances open up the principal possibility of constructing an automata model of the clinical process as an inference engine for CDSS.

A significant role in the clinical process is played by possible contradictions between the various types of medical activities offered to the patient. For example, the prescribed medication may worsen the course of another disease that the patient suffers from, may be contraindicated in connection with certain medical facts from the medical history, or may enter into undesirable interactions with other medicines that the patient is taking. The identification of such contradictions and their demonstration to the doctor is one of the tasks of the CDSS. In the case of multiple sclerosis, this problem is especially acute. Here, in order to achieve and maintain the desired effect, drug therapy should continue continuously and for a long time, and the existing range of drugs has a very wide range of adverse reactions up to complete intolerance.

The need for individual selection and flexible change of drugs prescribed for a particular patient poses the problem of making and verifying the current changes in the automata model used in the CDSS.

As emphasized in [Mashkoor], for modeling of medical devices, rigorous method are needed, and the notion of refinement plays the pivotal role here, supporting the correct-by-construction approach for development. Moreover, each refinement is accompanied by a verification stage, which guarantees secure operation of a medical device. It seems appropriate to extend these approaches to the development of automata models as inference engines in CDSS. To the best of our knowledge, there is no previous study that analyzes the possibilities of making and verifying the current changes in the automata model used in the CDSS.

Therefore, the article raises the following questions: 
- How to define a refinement in which the clinician can make flexible changes in CDSS states without the participation of an IT developer?

- How to ensure verification of such a refinement?

The further material is organized as follows. Section 3 discusses possibilities and conditions of implementation of flexible changes in an automata model. In section 4 the ways of verification of flexible change in the automatic model are under consideration. In section 5 as a case study implementation of an automata model with flexible change as an inference engine in CDSS for multiple sclerosis is considered.

\section{IMPLEMENTING OF FLEXIBLE CHANGES IN AN AUTOMATA MODEL}

Consider an example. Figure 1 shows a fragment of an automata modeling the clinical protocol for the treatment of multiple sclerosis, which includes the following standard states: Identification of exacerbation degree, SMT, Pulse therapy and result evaluation, Plasmophoresis (АСТH), Checking the type of course. In addition, according to the physician's opinion, in the case of a severe exacerbation, dexamethasone may be used instead of pulse therapy (the corresponding state Dexamethasone and result evaluation is shown in Figure 1 as optional).

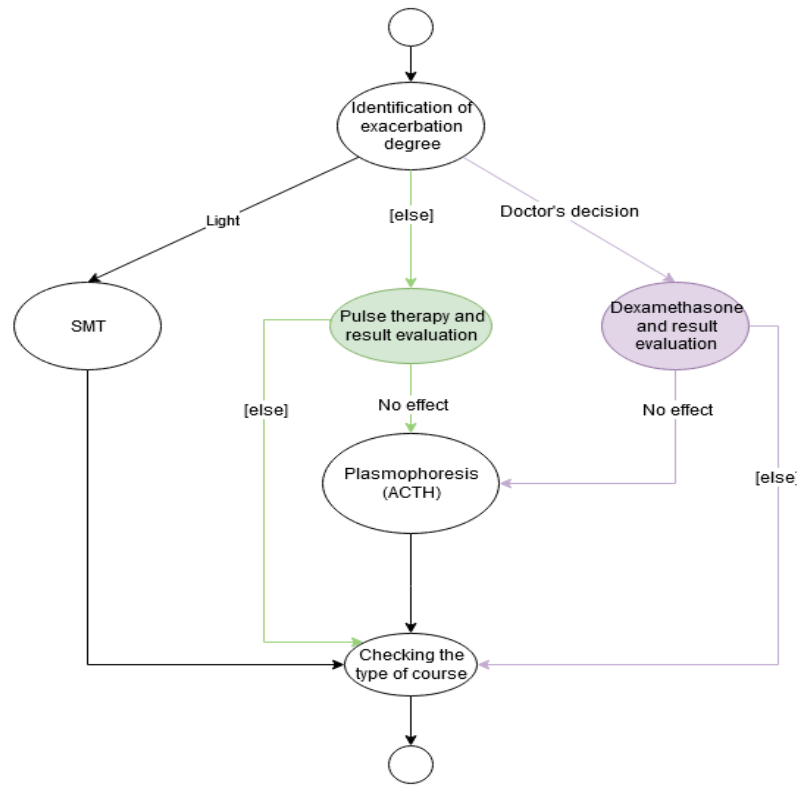

Figure 1. Fragment of a clinical protocol for the treatment of multiple sclerosis

However, in this case, the physician needs to check whether the use of dexamethasone is contraindicated in relation to all precedent and subsequent appointments and procedures, which are provided for by the further execution of the clinical protocol. A formal description of this problem is illustrated in Figure 2. Let the basic automata is in state A, state B called "standard" is responsible for fulfilling some medical activities (medicines and / or procedures), state $\mathrm{C}$ is the resulting one, state $\mathrm{X}$ can be added to basic automata optionally. Adding state $\mathrm{X}$ leads to the appearance of a new path from state $\mathrm{A}$ to state $\mathrm{C}$ through the new state $\mathrm{X}$.

Medical activities being performed in state B may conflict with medical activities performed in previous states (they are conditionally labeled as state D in Figure 2a). However, these contradictions are revealed during the verification of the basic automata, and the history of step-by-step verification is preserved. On this basis, when state $\mathrm{X}$ is added, possible contradictions of its medical activities with those of previous states can be detected automatically.

Consider now some state $\mathrm{E}$ (Figure $2 \mathrm{~b}$ ) attainable from state $\mathrm{C}$ (possibly through several transitions). Prior to adding state X, the automata was checked, respectively, states A, B, C, and E did not contradict each other, 
however, the added state $\mathrm{X}$ may contradict state $\mathrm{E}$, for example, due to contraindications between medicines in some states or due to some transitions non-availability. However, the possible contradictions of the medical activities of state $\mathrm{X}$ with the medical activities performed in subsequent state $\mathrm{E}$ cannot be predicted by the above method.

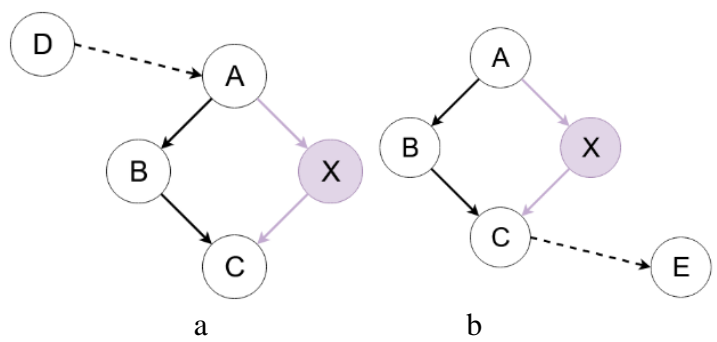

Figure 2. A formal model of a fragment of an automata with contradictions

To understand the conditions under which it is possible to add a new state $\mathrm{X}$ to an already existing automata model, we introduce the concepts of a "similar" state.

Let the medicine or procedure $\mathrm{Db}$ be used in state $\mathrm{B}$ with the contraindications $\mathrm{CDb} 1, \mathrm{CDb} 2, \ldots$ Let state $\mathrm{B}$ also have an input transition from state $\mathrm{A}$, and (possibly several) output transitions to states $\mathrm{C} 1, \mathrm{C} 2 \ldots$

Then let us call a "similar" state of state B, such state X that it uses medicine or procedure Dx with contraindications $\mathrm{CDx} 1, \mathrm{CDx} 2, \ldots$ and:

- there is an input transition from state $\mathrm{A}$ to state $\mathrm{X}$ and output transitions $\mathrm{C} 1, \mathrm{C} 2 \ldots$;

- there are as many exit transitions from state $\mathrm{X}$ as there are from state $\mathrm{B}$;

- there is a bijection (one-to-one correspondence) between each output transition from B and each output transition from $\mathrm{X}$.

The input transitions from state A to states B and X must have different conditions (to maintain general orthogonality). Since the transition to state $\mathrm{X}$ is performed by the doctor's decision, the condition for the transition to X is a predicate obtained as the logical "AND" conditions for the transition to B and the doctor's decision, which will provide the necessary orthogonality.

The transformation of the base state of an automata into a "similar" state can rightfully be considered as a transition in automata modeling from ground model to refinement. Thus, in order to get the source code describing the "similar" state X, it is enough to take the code describing the "standard" state B and change the attributes that are responsible for the parameters, namely the medicines and $\backslash$ or procedures and contraindications.

\section{VERIFICATION OF FLEXIBLE CHANGES IN THE AUTOMATA MODEL}

According to the review [Bonfanti], model checking [Emerson] dominates among verification methods for medical devices. The general limitations of the model checking method include the following: (1) Only models with a finite number of states are supported. (2) There is a state-space explosion problem characteristic of large multiprocessor systems. (3) Using the method, only a system model is checked instead of a real automatic program, and not any model properties, but only temporal ones. However, due to the specifics of the subject area of application of the automata model, namely the support of the clinical process in the presence of flexible changes, restrictions (1) and (2) are not fundamental.

The list of model-checking tools for medical devices is rather vast [Bonfanti]. However, in relation to the automata used as an inference engine in CDSS, the most effective was the use of the UniMod.Verifier, that works by integrating the UniMod tool [Gurov, Ricca] and the Bogor program verifier [Robby]. When using UniMod.Verifier, there is no need to convert the automatic program into the input language of the verifier. A significant advantage of UniMod.Verifier compared to other verifiers is that it uses the UniMod interpreter of automata systems. Therefore, UniMod.Verifier does not check the correct operation of the program model, but the automata system executed by the UniMod tool. This eliminates the above limitation (3) of the model checking method. 
Program requirements of the UniMod.Verifier are written in the temporal logic language LTL using the following operators: LTL.always (G); LTL.eventually (F); LTL.next (X); LTL.until (U); LTL.weakUntil (W), p W q = (p U q) $\| \mathrm{G}(\mathrm{p} \& \&$ !q); LTL.release (R, p R q = !(!p U !q); LTL.negation; LTL.equivalence; LTL.implication; LTL.conjunction; LTL.disjunction.

To verify the automata program in UniMod.Verifier, one first needs to create an XML description that is automatically executed in UniMod. Moreover, when using some other verifiers (for example, Convert), UniMod can also be used to save the automatic program in XML format.

Then one needs to write down the formula for verification. To do this, first the predicates are declared, and then the formula is written using these predicates as nested function calls. For example, a predicate with the name "no_effect" and the value AutomataModel.wasEvent (model, "CMT") is true when the last processed event is CMT.

The verifier is called by a special command, for example: verifier.cmd TestHospital.connectivity AfterDegreeofExacerbation,

where TestHospital.connectivity is the name of the description file of the automated program in UniMod format with the connectivity extension, and AfterDegreeofExacerbation is the name of the function for verification.

Let us compare the formula for verifying a fragment of the automata model presented in figure 1 written using the described formalisms. For ground model, i.e. using only "standard" states, the formula looks as follows:

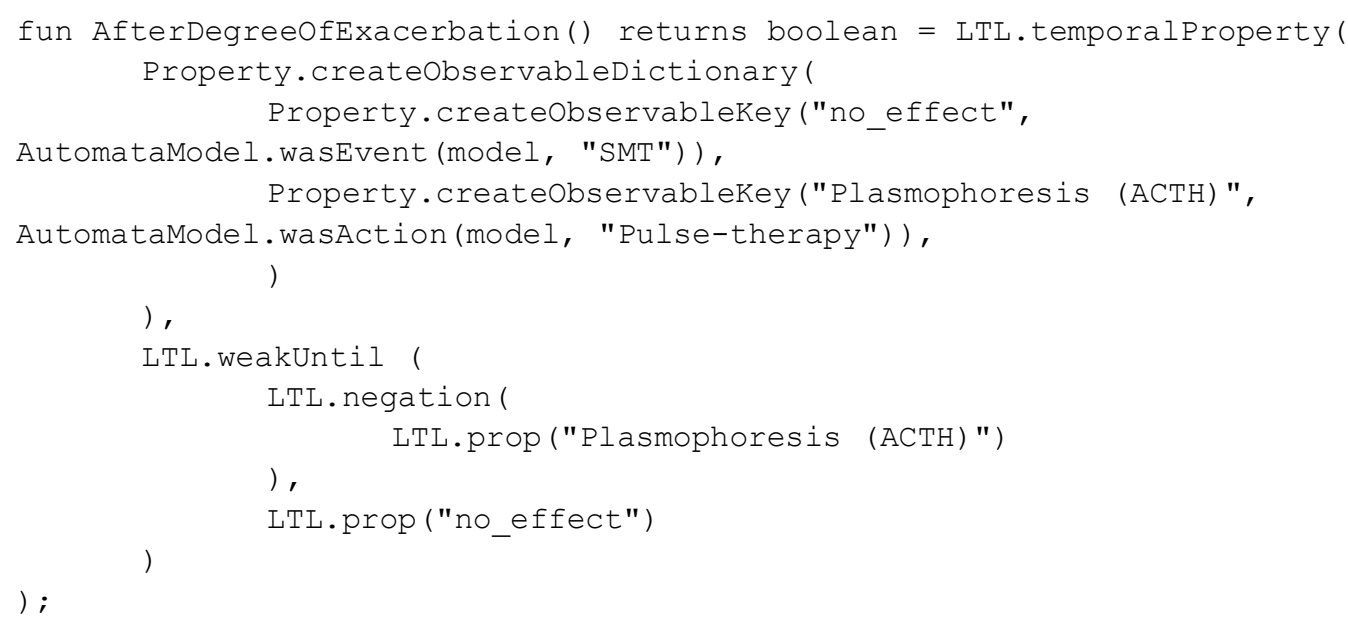

After calling the appropriate command, standard information about the verification process will be written to standard output. The result of the verification can be either a success message or an error message after verification in the form of a counterexample that describes the steps of the state of the machine, for example:

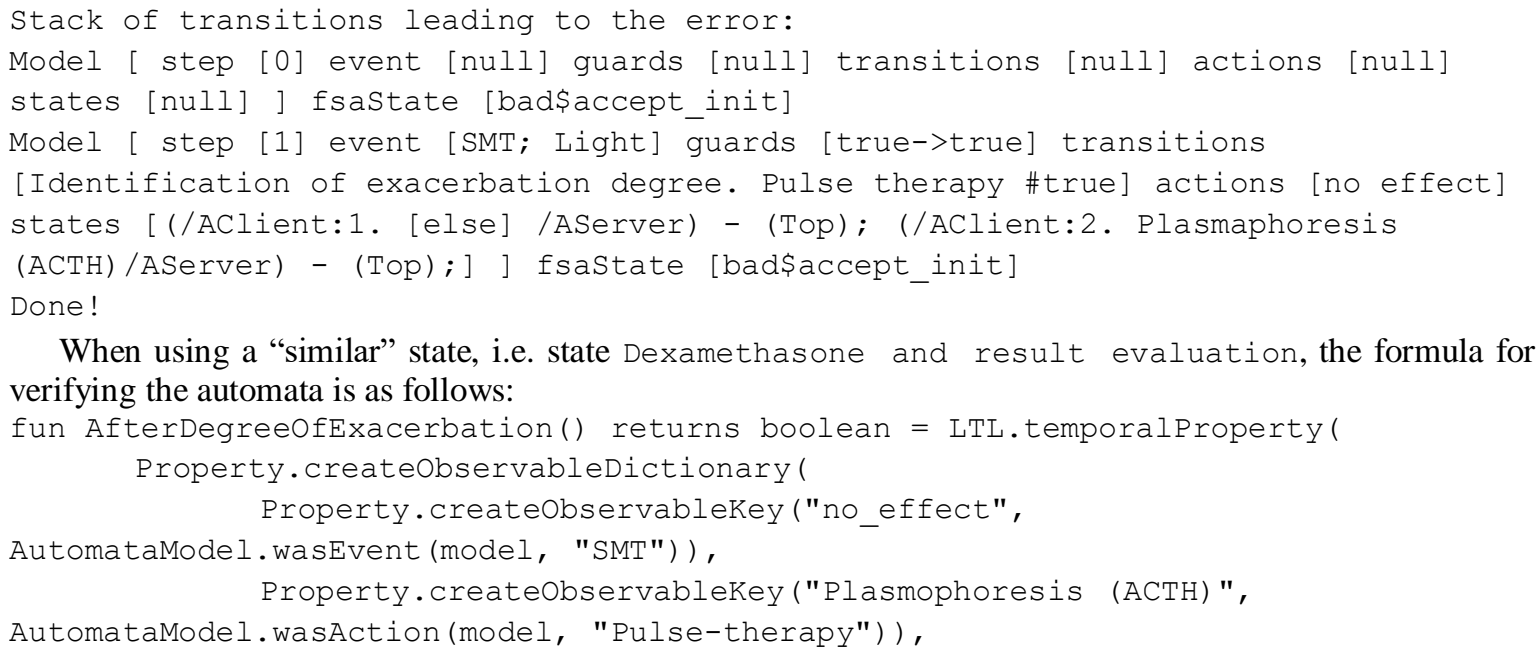

When using a "similar" state, i.e. state Dexamethasone and result evaluation, the formula for verifying the automata is as follows: 


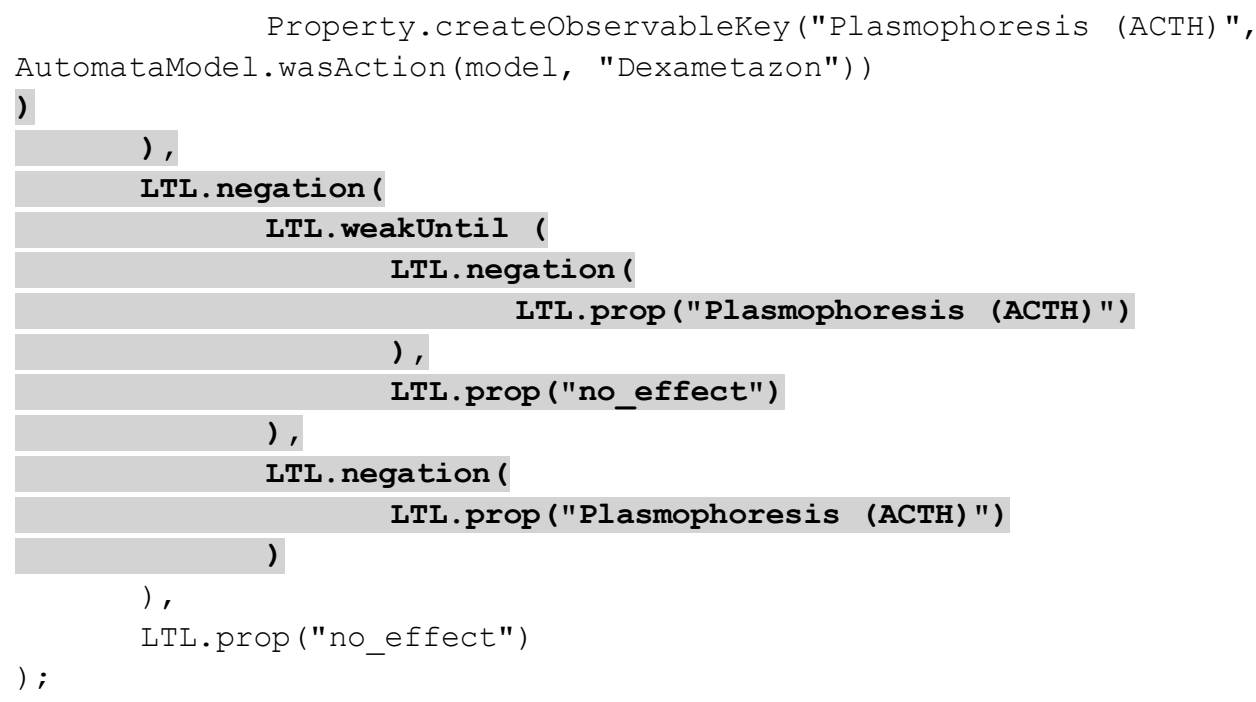

It is easy to see that in this case a transition is added after the Dexametazon state and in the automata model new conditions for the transition from previous states to the final one are added (the corresponding changes are highlighted in the code). For other possible contraindications that will appear in subsequent states, changes to the verification formula are to be made in a same way.

Thus, upon activation of a "similar" state, a pre-prepared verification code is launched that corresponds to all interactions with this state.

\section{IMPLEMENTATION OF AN AUTOMATA MODEL WITH FLEXIBLE CHANGE AS AN INFERENCE ENGINE IN CDSS FOR MULTIPLE SCLEROSIS}

The implemented CDSS is based on a combination of "design patterns + automata", which ensures compliance with the requirements of standardizing the clinical process both from the point of view of clinicians and from the point of view of IT developers. To work with the contradictions that arise during the clinical process, specialized design patterns are used in the architecture of the developed CDSS. The architecture diagram is shown in Figure 3.

For a flexible selection of various strategies of working with contradictions, the Strategy and Facade patterns are used. The IMedicalstrategy interface is implemented using specific strategies containing behavior for each of the scenarios of working with contradictions arising between various medical activities offered to the patient, including drugs and / or procedures. The logic of working with automata is hidden in the StateMachine class, which provides the setState (), getState (), and getAllstates () methods as an interface. To make clinical decisions, the attending physician should be provided with a quick and easy way to work with various sources of medical information. To do this, the Adapter pattern is foreseen, which allows to combine various data sources into a single interface for interaction and use them together. The UserInterfaceControl class represents the user interface with which the doctor will interact.

The whole complexity of working with an automata machine including resolving contradictions is hidden in ground model variant behind the AutomataFacade view, which is one of the points of work with the solution presented.

The presented scheme makes it easy to make refinements of the kind described above. Namely, to add a test for contraindications when introducing a new "similar" state, it is enough to add one class, called Verifier, whose responsibility will be the verification of a new change, described in section 4 . The scheme will change as follows (the changes are highlighted in green in Figure 3): a dependency on the Verifier class will be added to AutomataFacade, and the verify () method will delegate verification to this class. To add a new "similar" state, the addSimilarState() method appears in AutomataFacade, which calls the verify () method, and, if successful, adds the passed "similar" state to the automata so that it can be used in 
the clinical process. An element (for example, a button) is added to the user interface, allowing the doctor to add a "similar" state.

Thus, the developed CDSS architecture based on the proposed automata model allows us to expand the scenarios of work with possible contradictions between medical activities contained in the clinical protocol without changing the code structure.

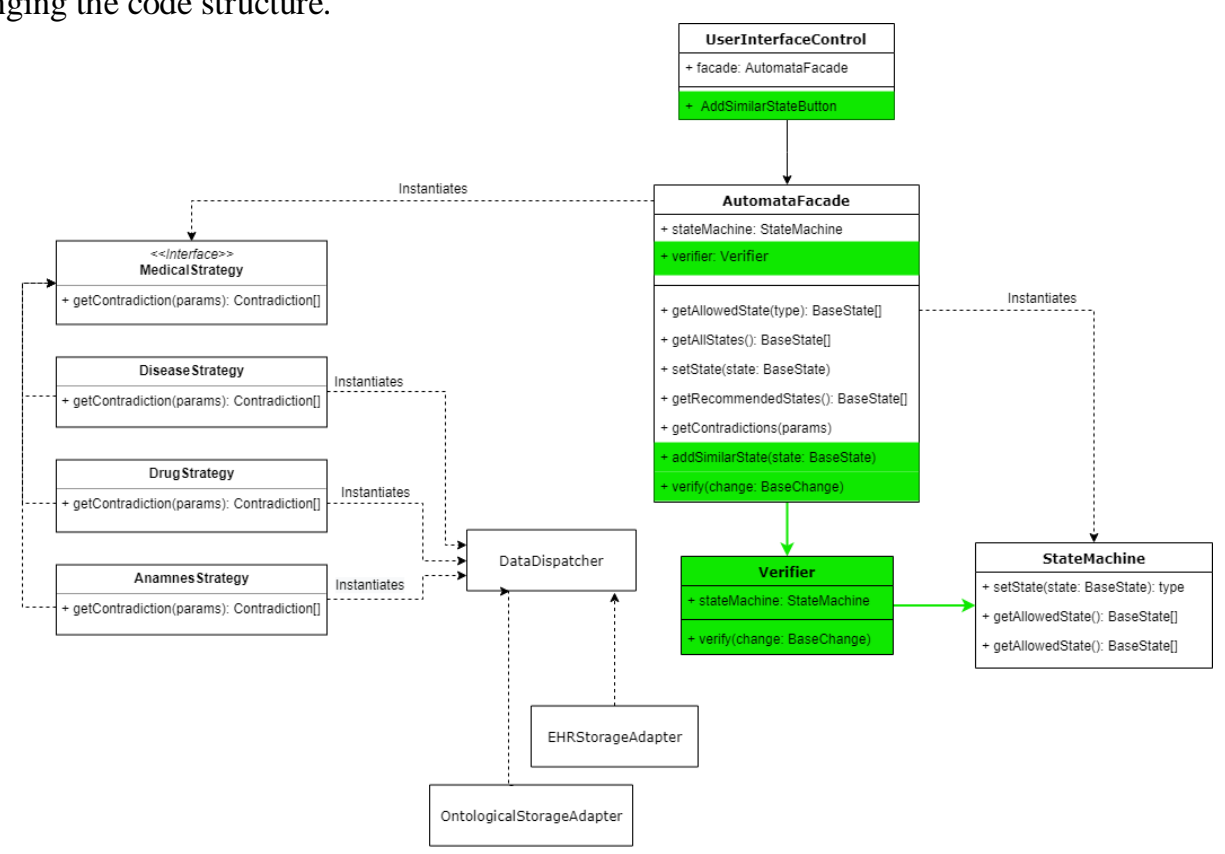

Figure 3. CDSS architecture diagram using design patterns

\section{CONCLUSION}

The following questions were posed in the article:

- How to define a refinement in which the clinician can make flexible changes in CDSS states without the participation of IT developer?

- How to ensure verification of such a refinement?

The answer to the first question was reduced to understanding the conditions under which it is possible to add a new state $\mathrm{X}$ to an already existing automata model. For this purpose the concepts of a "similar" state was introduced and defined. Thus, in order to get the source code describing the "similar" state X, it is enough to take the code describing the "standard" state B and change the attributes that are responsible for the parameters, namely the medicines and $\backslash$ or procedures and contraindications.

When answering the second question, it was shown that in relation to the automata used as an inference engine in CDSS, the most effective was the use of the UniMod. Verifier. Using a specific example of a fragment of an automata model to support the clinical process of multiple sclerosis, it was demonstrated that verification of refinement associated with the introduction of a "similar" state comes down to checking for possible contradictions between the parameters of medical activities in this state and in subsequent states. Thus, upon activation of a "similar" state, a pre-prepared verification code that corresponds to all interactions with this state can be launched without the help of IT-developer.

As a case study the implementation of CDSS for multiple sclerosis using an automata model as an inference engine in was depicted. It is shown that adding a "similar" state to the automata model requires very small changes in the CDSS scheme and can be called up and verified with a single interface button, i.e. this can be done by the doctor himself without the participation of an IT developer.

The further prospects of the work are associated with the expansion of the nomenclature and characteristics of possible online changes to the automata model in accordance with the specifics of other clinical processes. 


\section{ACKNOWLEDGEMENT}

This work was supported by Russian Science Foundation, Grant 19-19-00696.

\section{REFERENCES}

Banach R. Hemodialysis Machine in Hybrid Event-B //Abstract State Machines, Alloy, B, TLA, VDM, and Z. 5th Int.Conf., ABZ 2016, Linz, Austria, May 23-27, 2016. P. 376-393. (2016).

Bologva E.V., Prokusheva D.I., Krikunov A.V., Zvartau N.E., Kovalchuk S.V. Human-Computer Interaction in Electronic Medical Records: from the Perspectives of Physicians and Data Scientists // Procedia Computer Science. V.100. P. 915-920 (2016).

Bonfanti S., Gargantini A., Mashkoor A. A Systematic Literature Review of the Use of Formal Methods in Medical Software Systems. J. Softw. Evol. and Proc. 00:1-23 (2017).

Clinical Decision Support (CDS). Office of the National Coordinator for Health Information Technology. 2013. https://www.healthit.gov/policy-researchers-implementers/clinical-decision-support-cds, last access 31.08.2017.

Emerson A.E., Clarke E.M. Characterizing correctness properties of parallel programs using fixpoints. Automata, Languages and Programming, Lecture Notes in Computer Science, 85: 169-181 (1980)

Groot P., Hommersom A., Lucas P., Balser M., Schmitt J. Experiences in Quality Checking Medical Guidelines using Formal Methods. Proceedings Verification and Validation of Software Systems (VVSS 2007) March 23, 2007, Eindhoven, The Netherlands,, 2007; 164-78.

Gurov V.S., Mazin M.A. , Narvsky A.S., Shalyto A.A. UniMod: Method and Tool for Development of Reactive Object-Oriented Programs with Explicit States Emphasis /Proceedings of St. Petersburg IEEE Chapters. Year 2005. International Conference "110 Anniversary of Radio Invention”. SPb ETU "LETI". 2005. V. 2, pp. 106-110.

Gusarova N.F., Vatian A.S., Osipov S.V. Egorov N.V., Boitcov V.V., Ryngach E., Treshkur T., Tatarnikova A., Shalyto A. Retrieving and Processing Information from Clinical Algorithm via Formal Concept Analysis // Lecture Notes in Computer Science (including subseries Lecture Notes in Artificial Intelligence and Lecture Notes in Bioinformatics), 2019, Vol. 11871, pp. 525-534.

Horsky J., Schiff G.D., Johnston D., Mercincavage L., Bell D., Middleton B. Interface design principles for usable decision support: a targeted review of best practices for clinical prescribing interventions // J Biomed Inform. V. 45. P. 1202-1216 (2012).

Johansen M.L., O’Brien J.L. Decision making in nursing practice: A concept analysis. Nursing Forum. V. 51. P. 40-48 (2016).

Khoussainov B., Nerode A. Automata Theory and its Applications. Progress in Computer Science and Applied Logic, Volume 21. Birkhauser, 2001.

M'ery D, Singh N. Medical protocol diagnosis using formal methods. International Symposium on Foundations of Health Informatics Engineering and Systems - FHIES 2011, Lecture Notes in Computer Science, vol. 7151, Johannesburg, South Africa, 2012; 1-20, doi: 10.1007/978-3-642-32355-3 1.

Mashkoor A., Sametinger J. Rigorous Modeling and Analysis of Interoperable Medical Devices. MSM '16: Proceedings of the Modeling and Simulation in Medicine SymposiumApril 2016 Article No.: 5. Pages 1-8

Mitkin S. Automata-Based Programming in DRAKON Language // Programmnaya Ingeneria. 2019. V. 10. No. 1. P. 3-13. DOI: $10.17587 /$ prin.10.3-13

NINDS Multiple Sclerosis Information Page. National Institute of Neurological Disorders and Stroke. 18 Oct 2019. http://www.ninds.nih.gov/disorders/multiple_sclerosis/multiple_sclerosis.htm, last access 12.01.2020

openEHR Architecture. Architecture Overview. Revision 1.1. Pages: 87. Availible at: https://specifications.openehr.org/releases/1.0.1/architecture/overview.pdf, ac-cessed: 08.08.2019.

Polikarpova N. I., Shalyto A. A. Automata-based programming. SPb.: Piter. 2009 (rus)

Practice Guideline Recommendations Summary: Disease-modifying Therapies for Adults with Multiple Sclerosis. Recommendations from current practice guideline. Endorsed by the Consortium of Multiple Sclerosis Centers, the Multiple Sclerosis Association of America, and the National Multiple Sclerosis Society. April 2018. https://www.aan.com/Guidelines/home/GuidelineDetail/898, last access 12.01.2020 
Rawson T.M., Moore L.S.P., Hernandez B. et al. A systematic review of clinical decision support systems for antimicrobial management: are we failing to investigate these interventions appropriately? // Clinical Microbiology and Infection. Aug. 2017. V. 23. Is. 8. P. 524-532.

Reis W.C., Bonetti A.F., Bottacin W.E. et al. Impact on process results of clinical decision support systems (CПКPs) applied to medication use: overview of systematic reviews // Pharm Pract (Granada). Oct-Dec 2017. V.15 (4).

Ricca F., Leotta M., Reggio G., Tiso A., Guerrini G., Torchiano M. Using UniMod for Maintenance Tasks: An Experimental Assessment in the Context of Model Driven Development / Proceedings of 4-th International Workshop on Software Engineering (MISE), 2012. http://dx.doi.org/10.1109/MISE.2012.6226018

Robby D.M., Hatcliff J. Bogor: An Extensible and Highly-Modular Model Checking Framework, March 2003. // Proceedings of the Fourth Joint Meeting of the European Software Engineering Conference and ACM SIGSOFT Symposium on the Foundations of Software Engineering (ESEC/FSE 2003). Technical Report, SAnToS-TR2003-3.

Shalyto A.A. Software Automation Design: Algorithmization and Programming of Problems of Logical Control. Journal of Computer and Systems Sciences International. 2000. Vol.39. №6, p.899-916.

ten Teije A., Marcos M., Balser M., van Croonenborg J., Duelli C., van Harmelen F., Lucas P., Miksch S., Reif W., Rosenbrand K., Seyfang A. Improving medical protocols by formal methods. Artif Intell Med. 2006 Mar; 36(3): 193-209.

Tiffen J., Corbridge S.J., Slimmer, L. Enhancing clinical decision making: Development of a contentious definition \& framework. Journal of Professional Nursing. V. 30. P. 399-405 (2014).

Vatian A., Dudorov S., Ivchenko A., Smirnov K., Chikshova E., Lobantsev A., Parfenov V., Shalyto A., Gusarova N. Design Patterns for Personalization of Healthcare Process // ACM International Conference Proceeding Series. ICGDA 2019: Proceedings of the 2nd International Conference on Geoinformatics and Data Analysis 2019, pp. 83-88. 